\title{
Recovery of neurofilament following early monocular deprivation
}

\section{Timothy P. O'Leary, Matthew R. Kutcher, Donald E. Mitchell and Kevin R. Duffy*}

Department of Psychology, Life Sciences Centre, Dalhousie University, Halifax, NS, Canada

\section{Edited by:}

Andrew J. Parker, University of

Oxford, UK

Reviewed by:

Gregor Rainer, University of

Fribourg, Switzerland

Preston E. Garraghty, Indiana

University, USA

${ }^{*}$ Correspondence:

Kevin R. Duffy, Department of

Psychology/Neuroscience,

Dalhousie University, Halifax, NS

B3H 4R2, Canada.

e-mail:kevin.duffy@dal.ca
Postnatal development of the mammalian geniculostriate visual pathway is partly guided by visually driven activity. Disruption of normal visual input during certain critical periods can alter the structure of neurons, as well as their connections and functional properties. Within the layers of the dorsal lateral geniculate nucleus (dLGN), a brief early period of monocular deprivation can alter the structure and soma size of neurons within deprived-eye-receiving layers. This modification of structure is accompanied by a marked reduction in labeling for neurofilament protein, a principle component of the stable cytoskeleton. This study examined the extent of neurofilament recovery in monocularly deprived cats that either had their deprived eye opened (binocular recovery), or had the deprivation reversed to the fellow eye (reverse occlusion). The loss of neurofilament and the reduction of soma size caused by monocular deprivation were ameliorated equally and substantially in both recovery conditions after 8 days. The degree to which this recovery was dependent on visually driven activity was examined by placing monocularly deprived animals in complete darkness. Though monocularly deprived animals placed in darkness showed recovery of soma size in deprived layers, the manipulation catalyzed a loss of neurofilament labeling that extended to non-deprived layers as well. Overall, these results indicate that both recovery of soma size and neurofilament labeling is achieved by removal of the competitive disadvantage of the deprived eye. However, while the former occurred even in the absence of visually driven activity, recovery of neurofilament did not. The finding that a period of darkness produced an overall loss of neurofilament throughout the dLGN suggests that this experiential manipulation may cause the visual pathways to revert to an earlier more plastic developmental stage. It is possible that short periods of darkness could be incorporated as a component of therapeutic measures for treatment of deprivation-induced disorders such as amblyopia.

Keywords: neurofilament, cytoskeleton, monocular deprivation, reverse occlusion, dark-rearing, recovery, lateral geniculate nucleus, plasticity

\section{INTRODUCTION}

It is now well established that early in postnatal life the development of neurons in the geniculostriate visual pathway is partly guided by experience-driven activity. Disruption to normal vision during this time can alter the proper development of neurons and their connections, to the extent that visual function can become deeply and intractably impaired in cats (Wiesel and Hubel, 1963a; Dews and Wiesel, 1970; Giffin and Mitchell, 1978) and monkeys (LeVay et al., 1980; Harwerth et al., 1983). Although a multitude of physiological and anatomical changes occur after an early period spent in total darkness (complete visual deprivation), more pronounced alterations can follow a period of selected or biased visual exposure as is exemplified by unilateral visual deprivation where only one eye receives patterned visual input (Daw, 2006). Periods of such deprivation lasting only $6-24 \mathrm{~h}$ in cats have been shown to precipitate a physiological shift in the ocular preference of neurons so that the non-deprived eye comes to dominate visual responses (Wiesel and Hubel, 1963b; Olson and Freeman, 1980; Frank et al., 2001). Anatomical modification also results from a period of early monocular deprivation, and this is perhaps best demonstrated by the marked shrinkage of deprived neurons within the main relay structure of the primary visual pathway, the dorsal lateral geniculate nucleus (dLGN) of the thalamus (Wiesel and Hubel, 1963a; Guillery and Stelzner, 1970), and also by a reduction in the size of deprived eye ocular dominance columns in the visual cortex (Hubel et al., 1977; Kossut et al., 1983).

A large body of research now documents the temporal sequence of the many neural changes that follow a period of monocular deprivation. Modification of the gross structure of neurons in the dLGN (Wiesel and Hubel, 1963a; Guillery and Stelzner, 1970) is evident 4-5 days after deprivation onset (Duffy and Slusar, 2009), and certain changes such as the modification of geniculocortical afferents achieve asymptotic levels within a week (Antonini and Stryker, 1993). Much of what is known about the intracellular events that underlie changes in structure has been obtained from work on rodents. Within the first few days of unilateral occlusion in rats, neurons in the primary visual cortex 
exhibit a reduction in the number of synaptic contacts as a consequence of increased proteolytic activity (Mataga et al., 2004). During the period in which dendritic spines are modified there is an NMDA-receptor-mediated molecular cascade that causes significant depression of synaptic efficacy between deprived cells, which is evident within $24 \mathrm{~h}$ of deprivation (Heynen et al., 2003), and which presumably helps to explain how cortical cells become comparatively less responsive to stimulation of the deprived eye (Wiesel and Hubel, 1963b; Mioche and Singer, 1989). Though less is known about the cellular events that mediate the lateroccurring changes in gross structure of deprived neurons, the tight link between structural modification and alteration of the neuronal cytoskeleton suggests that the cytoskeleton provides a substrate upon which deprivation acts to produce gross changes in neuron structure (Kutcher and Duffy, 2007).

The neuron cytoskeleton comprises three protein classes: actin filaments, intermediate filaments, and microtubules. Proteins within the intermediate class form a stable and stationary intracellular scaffold that is well suited to provide gross structural stability (Morris and Lasek, 1982; Yuan et al., 2006). Evidence that intermediate filaments provide gross structural support of neurons has emerged from studies of neurofilament, an intermediate filament abundant in mature neurons (Morris and Lasek, 1982). The absence of axonal neurofilament leads to a reduction in radial growth of axons (Yamasaki et al., 1992; Sakaguchi et al., 1993), and the loss of neurofilament in neurons of monocularly deprived kittens has been linked in space and time with alterations in neuron gross structure (Kutcher and Duffy, 2007; Duffy and Slusar, 2009). Three neurofilament subunits assemble to form a protein heteropolymer, with each subunit named according to its molecular mass: light $(70 \mathrm{kDa})$, medium $(155 \mathrm{kDa})$, and heavy $(200 \mathrm{kDa})$. The characteristics of neurons in the dLGN labeled for each neurofilament subunit are strongly overlapping (Duffy et al., 2011).

Though the various effects of early monocular deprivation can be permanent, substantial recovery can occur provided that normal vision is restored to the deprived eye sufficiently early in postnatal life during so-called critical periods. Some restoration of visual function to the deprived eye can occur in such circumstances if the eye is either opened to provide binocular visual input (binocular recovery; BR), or the deprivation is reversed (reverse occlusion; RO) to force usage of the originally closed eye (Dews and Wiesel, 1970; Giffin and Mitchell, 1978; Movshon, 1976a), though recovery is reliably slightly better with reverse occlusion (Giffin and Mitchell, 1978). These behavioral regimes can also produce substantial physiological recovery in the visual cortex (Dürsteler et al., 1976; Movshon, 1976b; Mitchell et al., 1977; Olson and Freeman, 1978), and reverse occlusion can result in recovery of anatomical features (Dürsteler et al., 1976; Antonini and Stryker, 1998). Although the mechanisms that mediate recovery from the effects of monocular deprivation are at present poorly understood, the coincidence of gross structural modification in the dLGN and the loss of neurofilament after a period of monocular deprivation (Bickford et al., 1998; Duffy et al., 2007; Kutcher and Duffy, 2007; Duffy and Slusar, 2009) led us to explore whether neurofilament is ameliorable and whether it might be involved in the recovery that can follow from early monocular deprivation. To explore this issue further, we examined the temporal changes of labeling for the commonly studied heavy neurofilament subunit (NF-H) in the dLGN of monocularly deprived kittens under rearing conditions of reverse occlusion and binocular recovery.

The results of our investigation show that NF-H labeling is recovered in neurons within visually deprived layers of the dLGN following either reverse occlusion or binocular recovery. Recovery required the removal of the competitive disadvantage of the deprived eye together with the presence of visually driven activity from this eye. As instructive as these results were, a potentially more important finding was obtained from animals that were placed in complete darkness following monocular deprivation. The finding that dark-rearing catalyzed a loss of neurofilament throughout the dLGN highlights a potential therapeutic effect of a period of darkness imposed early in postnatal life.

\section{MATERIALS AND METHODS ANIMALS AND REARING CONDITIONS}

Recovery from the effects of early monocular deprivation was examined in the dLGN of 23 kittens that were bred and reared in a closed animal colony. The breeding, rearing, and experimental procedures followed protocols approved by the Dalhousie University Committee on Laboratory Animal Care following guidelines established by the Canadian Council on Animal Care. Monocular deprivation was begun at postnatal day 34, at about the peak of the sensitive period for ocular dominance plasticity (Olson and Freeman, 1980), and was maintained for 7 days. Monocular deprivation was then relieved and animals were thereafter reared in one of two recovery conditions: reverse occlusion $(N=6)$ or binocular recovery $(N=6)$. Recovery was maintained for durations of 1,4 , or 8 days; with two animals examined at each recovery duration (Table $\mathbf{1}$ ). The extent to which recovery depended upon visually driven experience was examined in 10 monocularly deprived cats that were placed in a light-tight dark room facility (Beaver et al., 1993) and were dark reared for either $1(N=2), 4(N=4)$ or $8(N=4)$ days. The dLGN of one normal animal at postnatal day 40 was also examined.

Monocular deprivation was achieved by closing the lids of the left eye using general anesthesia (1-3\% isoflurane in oxygen) following procedures described by Murphy and Mitchell (1987). The upper and lower palpebral conjunctivae of the eye were sutured together using 4-0 Ethicon vicryl and the lid margins were left unaltered to enable normal use of the lids following the period of deprivation. After 7 days, the deprivation was ended by removal of the sutures to restore binocular visual input (binocular recovery), or by opening of the deprived eye and closing of the eyelids of the fellow eye (reverse occlusion). All animals were given post-operative analgesics and antibiotics according to protocol guidelines.

\section{HISTOLOGY}

Cats were injected with a lethal dose of Euthanyl $(150 \mathrm{mg} / \mathrm{kg})$, and were exsanguinated by transcardial perfusion with $200 \mathrm{ml}$ of phosphate buffered saline (PBS; $4^{\circ} \mathrm{C}$; $\mathrm{pH} 7.4$ ), and then with $200 \mathrm{ml}$ of $4 \%$ paraformaldehyde dissolved in PBS ( $4^{\circ} \mathrm{C}$; $\left.\mathrm{pH} 7.4\right)$. The cerebral hemispheres were resected from the underlying 
Table 1 | Measurements of cross-sectional soma area and NF-H immunoreactive cell density in initially non-deprived and initially deprived layers of the dLGN for all the animals examined in this study.

\begin{tabular}{|c|c|c|c|c|c|}
\hline Animal \# & Condition & $\begin{array}{l}\text { Initially non-deprived } \\
\text { soma area }\left(\mu \mathrm{m}^{2}\right)\end{array}$ & $\begin{array}{l}\text { Initially deprived } \\
\text { soma area }\left(\mu \mathrm{m}^{2}\right)\end{array}$ & $\begin{array}{l}\text { Initially non-deprived } \\
\text { NF-H density (neurons } / \mathrm{mm}^{2} \text { ) }\end{array}$ & $\begin{array}{l}\text { Initially deprived NF-H } \\
\text { density (neurons } / \mathrm{mm}^{2} \text { ) }\end{array}$ \\
\hline C160 & Normal & 215 & 202 & 99 & 91 \\
\hline C019 & 1 day $\mathrm{RO}$ & 232 & 184 & 50 & 31 \\
\hline C015 & 4 day $\mathrm{RO}$ & 220 & 195 & 54 & 37 \\
\hline C018 & 8 day $\mathrm{RO}$ & 177 & 204 & 43 & 59 \\
\hline $\mathrm{C} 010$ & 1 day BR & 266 & 216 & 58 & 28 \\
\hline C011 & 1 day BR & 314 & 228 & 35 & 14 \\
\hline $\mathrm{C} 007$ & 4 day $B R$ & 226 & 207 & 28 & 15 \\
\hline C008 & 4 day BR & 290 & 246 & 28 & 18 \\
\hline $\mathrm{C} 023$ & 4 day DR & 217 & 188 & 51 & 36 \\
\hline $\mathrm{C} 021$ & 4 day DR & 222 & 201 & 36 & 27 \\
\hline C131 & 4 day DR & 198 & 174 & 12 & 6 \\
\hline $\mathrm{C} 133$ & 4 day DR & 187 & 183 & 27 & 16 \\
\hline $\mathrm{C} 022$ & 8 day DR & 209 & 195 & 25 & 25 \\
\hline $\mathrm{CO} 20$ & 8 day DR & 220 & 205 & 28 & 24 \\
\hline $\mathrm{C} 134$ & 8 day DR & 197 & 189 & 3 & 1 \\
\hline C135 & 8 day DR & 210 & 205 & 7 & 5 \\
\hline
\end{tabular}

For convenience, the right A layer and the left A1 layer of the dLGN were designated as "initially deprived" for the normal animal (C160).

thalamus and all tissues were placed in PBS $(0.1 \mathrm{M}, \mathrm{pH} 7.4)$ containing $30 \%$ sucrose and left floating at $4^{\circ} \mathrm{C}$ for $48 \mathrm{~h}$ to cryoprotect for sectioning. Coronal slices of the dLGN were sectioned at a thickness of $50 \mu \mathrm{m}$ using a freezing microtome (American Optical Corporation, South-bridge, MA). Sections of the LGN were stained for Nissl substance, and adjacent sections were labeled for NF-H. Sections destined for Nissl staining were mounted onto gelatin-coated glass slides and were air-dried overnight. Sections were dehydrated in a graded series of ethanol concentrations and submerged in cresyl violet solution $(0.1 \%)$ for $5 \mathrm{~min}$, and were then differentiated in ethanol concentrations to optimize the staining contrast between perikarya and background. Tissue was then cleared in Histo-Clear (National Diagnostics, Atlanta, GA) and coverslipped with Entellan mounting medium (EMD Chemical, Darmstadt, Germany).

Neurofilament protein was made visible by labeling with a monoclonal antibody, SMI-32 (Convance Research Products, USA) that targets a non-phosphorylated epitope of the heavy molecular weight neurofilament subunit. An examination of antibody specificity with immunoblots of cat visual cortex produced a single reacted band with an apparent molecular mass of approximately $130 \mathrm{kDa}$, a finding consistent with previous studies of nonphosphorylated NF-H (Julien and Mushynski, 1982; Kaufmann et al., 1984; Georges and Mushynski, 1987). Free-floating sections were washed in a solution of PBS and 5\% normal goat serum for 1h. Sections were then incubated overnight in PBS with 5\% normal goat serum and SMI-32 antibody (1:1000). Immunolabeling was rendered visible by use of a Vectastain $A B C$ kit (Vector Laboratories, Burlingame, CA) and the 3,3'-diaminobenzidine tetrahydrochloride chromogen. Reacted sections were mounted onto glass slides, dehydrated in ethanol, cleared in Histo-Clear and coverslipped with Entellan.

All images of antibody labeling were arranged into figures using Photoshop CS3, and the "levels" tool was used to make slight adjustments to the overall contrast of images for consistency between figures.

\section{QUANTIFICATION}

Cross-sectional soma area of neurons stained for Nissl substance was measured from the A laminae of the left and right dLGN cut through approximately Sanderson (1971) coronal plane 6-7, which is positioned at about the midpoint of the anterior-posterior axis. A computerized stereology system (newCAST; VisioPharm, Denmark) was used to randomly sample $50 \%$ of the area within the binocular region of layer A and of layer A1 separately at 1000 times magnification using a BX51 compound microscope fitted with a high-resolution DP-70 digital camera (Olympus, Markham, Canada). The cross-sectional area of neuron somata was measured from these sampled regions of dLGN using the nucleator stereology probe (newCAST; 
VisioPharm, Denmark). Neurons were distinguished from glial cells by selecting for measurement only those cells that showed a staining pattern congruent with neurons: dark cytoplasm, pale nucleus and dark nucleolus. Cells that did not match these criteria were not included in our sample. Area measurements were obtained from two sections that were separated by at least $100 \mu \mathrm{m}$ to avoid taking more than one measurement per neuron. The number of neurons measured per layer from each hemisphere ranged from approximately $20-100$, with averages of 50 neurons measured per layer from each hemisphere, and 200 neurons measured per cat. A deprivation metric (1) was used to measure the within-animal effect of providing a period of binocular vision or reverse occlusion after monocular deprivation. In the normal animal that we examined, layer A from the right dLGN and layer A1 from the left dLGN were designated as initially deprived. Normal animals have been shown to have a deprivation index that is approximately 0 (Kutcher and Duffy, 2007; Duffy and Slusar, 2009). Similar metrics have been used in the past to evaluate the effect of deprivation within the dLGN and cortex (Fava et al., 1999; Sato and Stryker, 2008).

\section{Deprivation Metric:}

$$
\begin{aligned}
& \text { (Non-Deprived A + Non-Deprived A1) } \\
& =\frac{-(\text { Deprived } A+\text { Deprived } A 1)}{(\text { Non-Deprived } A+\text { Non-Deprived } A 1)} \times 100
\end{aligned}
$$

Neurons that were labeled for NF-H were counted in the binocular region of layer A and A1. Neurons were counted only if they contained strong cytoplasmic labeling but weak nuclear labeling, which together ensured that only neurons cut through the somal midline were counted, and which also ensured that caps of neurons were excluded from our sample. Counts were completed with a stereology program (newCAST; VisioPharm, Denmark) that enabled use of the optical dissector probe. Counts were made at 1000 times magnification using a compound microscope fitted with a high-resolution digital camera (Infinity3-1M; Lumenera, Canada). A guard depth of $5 \mu \mathrm{m}$ was used for counts, and a total of $50 \%$ of each layer of the dLGN was sampled for counting. Immunopositive cell counts were made from two sections of each dLGN hemisphere that were separated by at least $100 \mu \mathrm{m}$. The density of neurofilament-positive cells was calculated for each layer by dividing the total number of counted cells by the size of the region sampled. For each animal, a deprivation metric was calculated for neurofilament positive cell density using the above Equation (1).

\section{STATISTICAL ANALYSES}

One-Way ANOVAs were completed separately for each rearing condition to determine if deprivation indexes for neurofilament positive cell density and cell soma size decreased across durations of recovery. Subsequent post-hoc comparisons were completed with one-tailed $t$-tests to determine at which recovery duration indexes were significantly different. As in other anatomical studies of the dLGN (e.g., Dürsteler et al., 1976; Garey and Vital-Durand, 1981; Tremain and Ikeda, 1982), One-Way ANOVAs were also completed for each animal separately to determine if the size of cells from deprived and non-deprived layers were significantly different.

\section{RESULTS}

Sections from the dLGN of all kittens reacted for NF-H showed labeling in cell bodies and dendrites that was distinct from a light background. Our measurements of NF-H labeling and soma size from the dLGN of one normal postnatal day 40 kitten (Table 1, C160) was in agreement with published reports (Bickford et al., 1998; Duffy et al., 2011), namely that A laminae serving the left and right eye contained about an equivalent number of NF-H-positive neurons, and that the size of neurons between eye-specific layers was comparable. As a baseline comparison for the recovery data, monocular deprivation for 7 days at the peak of the critical period has previously been shown to reduce the number of NF-H immunoreactive neurons in deprived eyerecipient laminae by $\sim 40 \%$, and their soma size by $\sim 25 \%$ smaller than non-deprived neurons (Kutcher and Duffy, 2007; Duffy and Slusar, 2009).

\section{REVERSE OCCLUSION}

Neurofilament-positive cell density and soma size were measured from the dLGN of cats that were reverse occluded for either 1, 4, or 8 days following a 7 day period of monocular deprivation. The effect of monocular deprivation on NF-H labeling was still clearly evident following 1 day of RO (Figure 1A): initially deprived layers (indicated with asterisks) contained only a smattering of immunopositive cell bodies and processes compared to initially non-deprived layers, which at this stage showed no evidence of a deprivation effect. Following 4 days of RO there was an obvious increase in the number of cells reactive for NF-H in initially deprived layers, but newly deprived layers retained many labeled cells and did not exhibit obvious signs of deprivation (Figure 1B). We inferred from this result that recovery of NF-H, which had become evident after 4 days of RO, occurred slightly before the loss of NF-H in the newly deprived layers. By 8 days of RO the loss of neurofilament reversed completely, with the initially non-deprived layers having far fewer neurons labeled for NF-H than the initially deprived layers, which at this stage manifested normal-appearing labeling (Figure 1C). Our measurements of neuron cross-sectional area revealed that the effect of RO on cell size occurred in synchrony with changes in NF-H (Figure1D). Quantitative measurements of the loss of NF-H density (average $=43 \%$ ) and the change in neuron size (average $=21 \%$ ) measured after 1 day of RO were considerably reduced after 4 days of RO (average $=29 \%$ and $13 \%$, respectively), and were both fully reversed when $\mathrm{RO}$ was extended to 8 days. Following 8 days of RO, originally deprived layers contained on average $32 \%$ more neurons labeled for NF-H than originally non-deprived layers, and originally deprived neurons were on average $17.5 \%$ larger than originally non-deprived neurons. An ANOVA performed on cell size measurements indicated that deprivation indexes decreased significantly over time $\left(F_{(2,3)}=176.39, p<0.001\right)$. The large number of cell size measurements obtained for each animal enabled us to perform ANOVAs for each animal separately, and this revealed that neurons from originally deprived layers were significantly smaller than neurons within originally non-deprived 


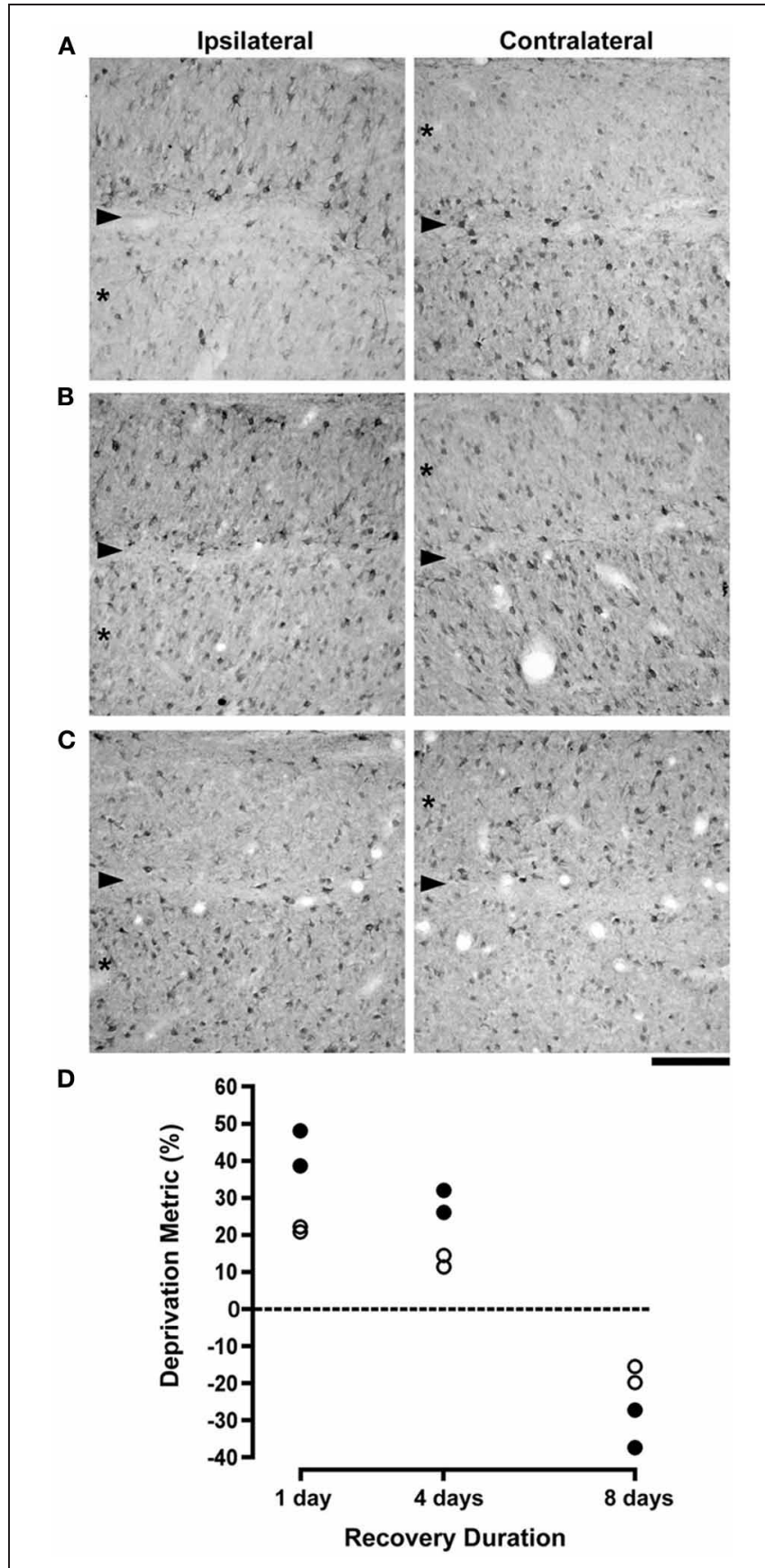

FIGURE 1 | The effect of different durations of RO on NF-H labeling and on neuron size in the A laminae of the dLGN. Photomicrographs in (A-C) show NF-H labeling in the left and right dLGN (respectively, ipsilateral and contralateral to the deprived eye) where the originally deprived layer in each image is indicated by an asterisk, and the boundary between A layers is identified with an arrowhead. The effect of a preceding week-long period of monocular deprivation was still clearly evident in the originally deprived layers of the dLGN after RO for 1 day (A). Following 4 days of $R O$ there was a recovery of NF-H labeling in originally deprived layers, but the originally non-deprived layers continued to show normal-looking NF-H labeling (B). By 8 days of $\mathrm{RO}$ there was a complete reversal of the deprivation effect so that originally deprived layers showed normal levels of NF-H labeling while labeling in the originally non-deprived layers was substantially reduced (C). Assessment of the recovery of neurofilament-positive cell density and

(Continued)

\section{FIGURE 1 | Continued}

neuron size in the dLGN was assessed with a deprivation metric (see Methods) that revealed a marked reversal of both NF-H labeling and neuron size. The size of neurons in originally deprived layers, which were smaller than originally non-deprived neurons at 1 day of $\mathrm{RO}$, were larger than originally non-deprived neurons by almost the same amount following 8 days of $\mathrm{RO}$ (open circles in $\mathbf{D}$ ). Likewise, originally deprived layers of the dLGN that exhibited comparatively fewer NF-H immunopositive neurons after 1 day of RO, contained considerably more NF-H labeled neurons following 8 days of $\mathrm{RO}$ (solid circles in $\mathbf{D}$ ). Therefore, $\mathrm{RO}$ for 8 days fully reversed the effect of monocular deprivation on neuron size and NF-H labeling. The dashed line in (D) represents the point at which no difference exists between measurements from right and left eye layers. Scale bar $=$ $100 \mu \mathrm{m}$.

layers for all cats following $1(p<0.0001)$ and 4 days of recovery $(p<0.005)$, but neurons from originally deprived layers were larger than originally non-deprived layers for all cats after 8 days of recovery $(p<0.005)$. An ANOVA performed on measurements from dLGN sections labeled for NF-H indicated that deprivation indexes decreased significantly over time $\left(F_{(2,3)}=\right.$ 86.22, $p<0.005)$, as deprivation indexes at 1 and 4 days were significantly larger than at 8 days of recovery (Figure 1D).

\section{BINOCULAR RECOVERY}

The effects of monocular deprivation were still readily apparent in animals whose originally deprived eye was opened for 1 day (Figure 2A). Compared to non-deprived layers, which contained many neurons labeled for NF-H, after 1 day of vision the originally deprived layers had many fewer immunopositive neurons and showed no evidence of recovery. Following 4 days of binocular vision there was an increase in the number of immunoreactive neurons in originally deprived layers but the deprivation effect was still obvious (Figure 2B). The effect of monocular deprivation on NF-H labeling was not evident after 8 days of binocular recovery, to the extent that the originally deprived layers were indistinguishable from non-deprived layers (Figure 2C). Our quantification of neuron size and labeling for $\mathrm{NF}-\mathrm{H}$ revealed evidence of a full recovery (Figure 2D). Following 1 day of binocular vision, there was on average $55 \%$ fewer neurons labeled for NF-H in originally deprived dLGN layers as compared to non-deprived layers, and originally deprived neurons were on average $23 \%$ smaller than non-deprived neurons. The difference between originally deprived and non-deprived neurons decreased as the duration of binocular vision extended to 4 days, and was reduced even further after 8 days. Following 4 days of binocular vision the difference in the comparative size of deprived neurons decreased to an average of $11.5 \%$, and was decreased further after 8 days to an average of $4.5 \%$, which is within the normal range. The density of NF-H labeled neurons after 4 days of binocular vision was on average $41 \%$ lower in deprived layers, and after 8 days was reduced to an average of $8 \%$, which was within normal limits. Statistical analysis of neuron size revealed that an ANOVA $\left(F_{(2,3)}=7.82, p=0.064\right)$ was only marginally significant, but post-hoc tests showed a significantly larger deprivation index at 1 day when compared to 8 days $(p<0.05)$. ANOVAs completed separately for each cat revealed that neurons from 


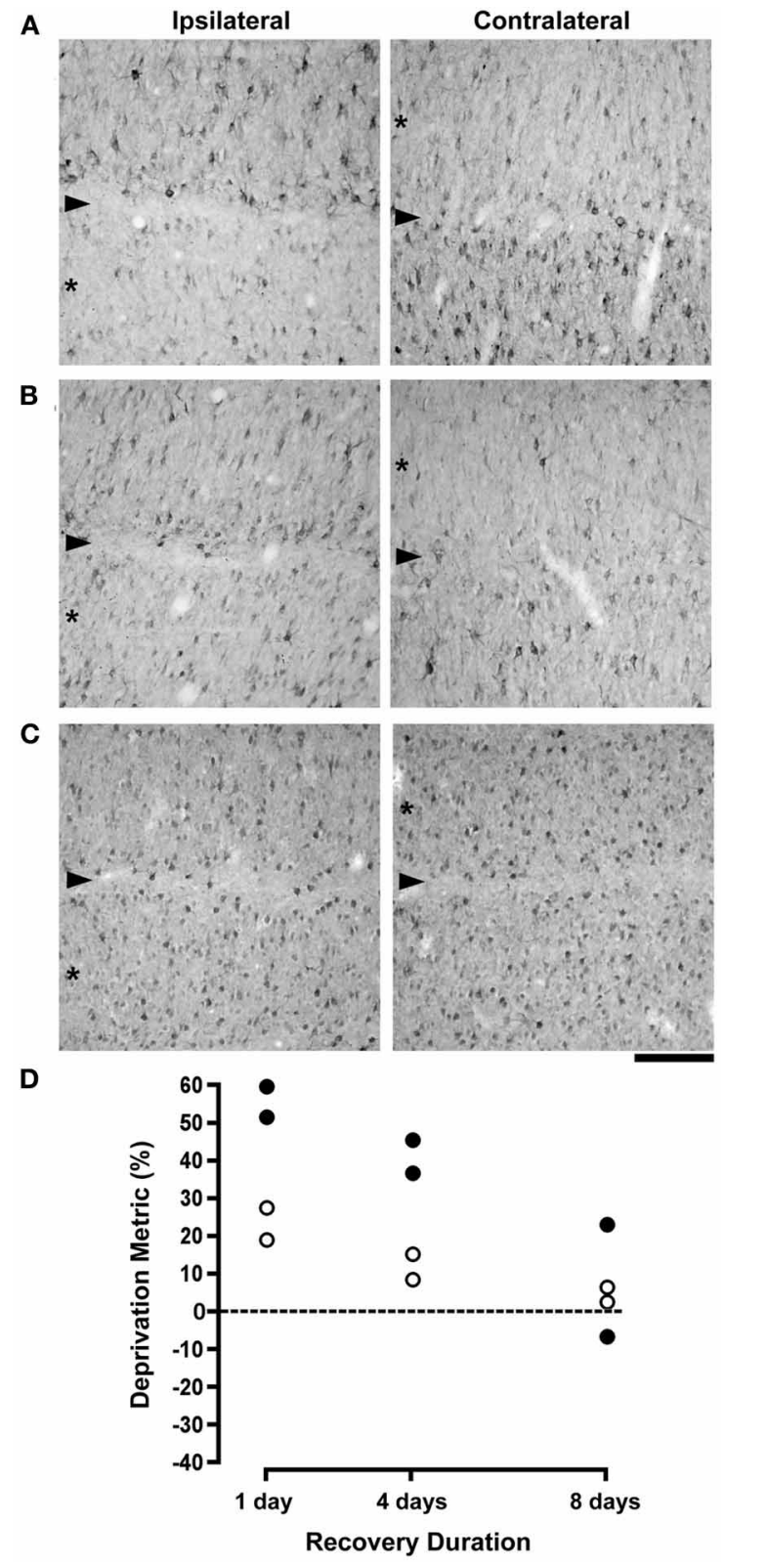

FIGURE 2 | The effects of different durations of binocular recovery following monocular deprivation are shown. Images in (A-C) show $\mathrm{NF}-\mathrm{H}$ labeling in the (A) laminae of the left (ispilateral to the deprived eye) and right (contralateral to the deprived eye) dLGN, with originally deprived layers indicated by asterisks, and the boundary between layers identified with an arrowhead. Labeling for NF-H remained substantially reduced in deprived layers after providing binocular vision for 1 day, indicating that there was little if any recovery with this small duration of binocular exposure (A). Subsequent to 4 days of binocular vision, the effect of monocular deprivation was still evident but noticeably reduced, as deprived layers showed the beginning of a recovery of NF-H labeling (B). Labeling for $\mathrm{NF}-\mathrm{H}$ in deprived layers appeared fully normal and indistinguishable from non-deprived layers after 8 days of binocular recovery (C). Results from the deprivation metric are plotted in (D), which reveals a gradual recovery of neuron size and NF-H labeling that was virtually complete following 8 days of binocular recovery. The dashed line in (D) represents the point at which no difference exists between measurements from right and left eye layers Scale bar $=100 \mu \mathrm{m}$. originally deprived layers were significantly smaller than neurons from non-deprived layers for all cats after $1(p<0.0001)$ and $4(p<0.05)$ days of recovery, whereas no difference was found in cats given 8 days of recovery. Statistics for NF-H density also showed a marginally significant $\operatorname{ANOVA}\left(F_{(2,3)}=6.87\right.$, $p=0.076)$, but again post-hoc tests revealed a significantly larger deprivation index at 1 day than at 8 days of binocular recovery $(p<0.05)$.

\section{DARK-REARING}

Results from the above studies provided evidence that the effect of monocular deprivation on NF-H labeling and neuron size was remediated by reverse occlusion or after restoration of binocular vision. Results from these studies suggest that removal of the competitive disadvantage of the deprived eye is sufficient to promote recovery; however, it is unclear whether the absence of a competitive disadvantage is sufficient by itself or whether the observed recovery additionally requires the presence of visually driven neural activity. We examined this issue by placing monocularly deprived animals into complete darkness, which simultaneously eliminated visual competition between the eyes while introducing balanced non-visually driven neural activity. Dark exposure also removed any benefit that visually driven activity may have had on recovery. We reasoned that placing a monocularly deprived animal in complete darkness could result in three possible outcomes for NF-H labeling: (1) the effects of monocular deprivation would persist unabated, which would indicate that recovery was dependent on visually driven activity; (2) the deprived layers would exhibit recovery, which would indicate that recovery while dependent on removal of the deprived eye's competitive disadvantage was not dependent upon visually driven activity; or, (3) the non-deprived layers would lose NF-H labeling and appear similar to labeling in deprived layers, a result that would indicate that removal of the competitive disadvantage alone is insufficient, and that in addition, visually driven activity is required to maintain a normal level of NF-H in the kitten dLGN. The results were in accord with the latter prediction.

Following 1 day of dark-rearing the effect of monocular deprivation on NF-H labeling remained obvious; the originally deprived layers had many fewer immunopositive cells than originally non-deprived layers, which appeared at this stage to be similar to normal (Figure 3A). However, when dark-rearing was extended to 8 days the results were quite different as there was a considerable decrease in immunolabeling in all A layers of the dLGN (Figure 3B). These qualitative observations were supported by quantification of NF-H labeling which revealed a considerable reduction in the number of neurons labeled for NF-H (Figure 3C) after 4 days of darkness. Following 8 days of dark-rearing the overall reduction in the density of NF-H neurons increased so that the effect of monocular deprivation was no longer evident. To determine if the decrease in NF-H labeling was significant, One-Way ANOVAs were completed separately for non-deprived and deprived layers using the mean NF-H density of cats at each recovery duration. Densities in both non-deprived $\left(F_{(2,7)}=19.37, p<0.005\right)$ and deprived layers $\left(F_{(2,7)}=6.34\right.$, $p<0.05)$ were significantly lower, as neurofilament density was greater after 1 day than after 4 and 8 days of dark-rearing. Our 

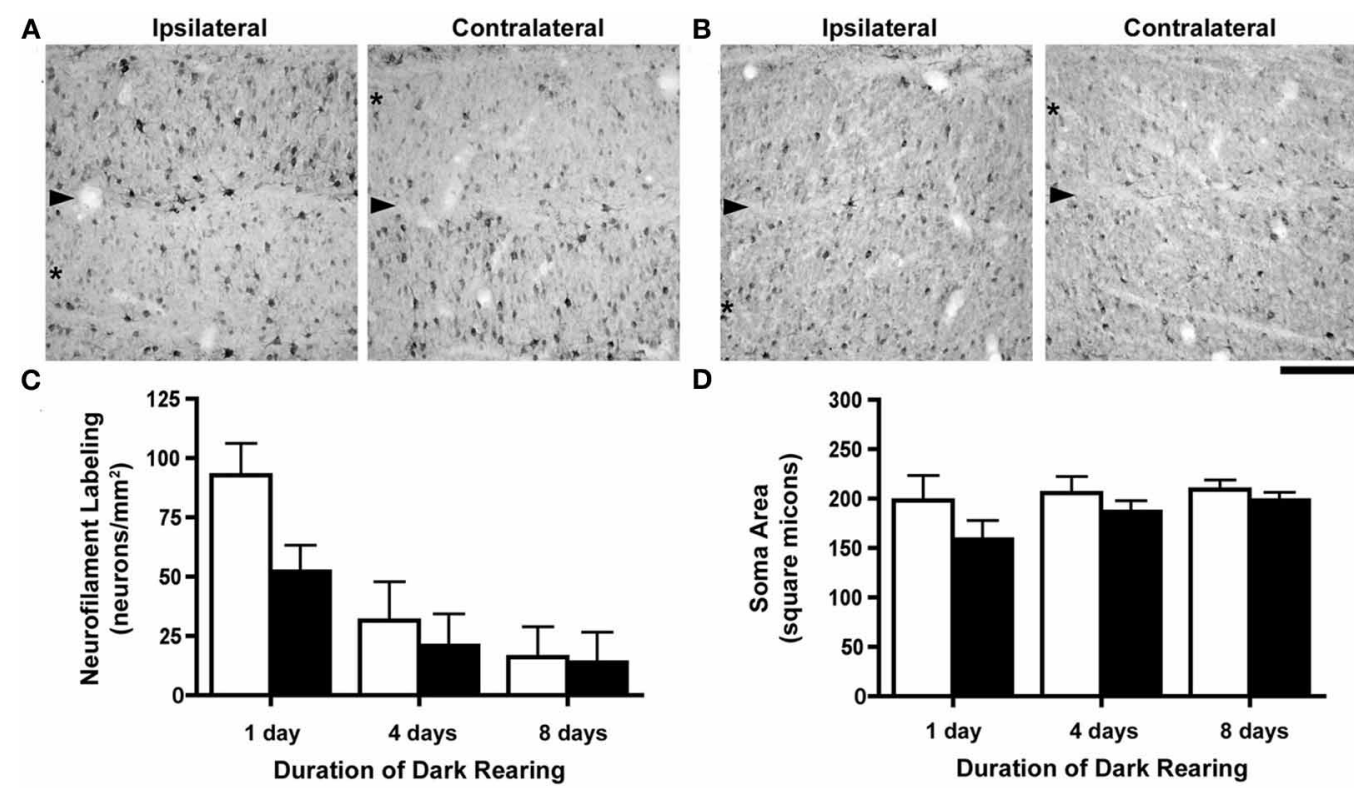

FIGURE 3 | The consequence of different durations of dark-rearing following a week-long period of monocular deprivation.

Images of the left (ipsilateral to the deprived eye) and right (contralateral to the deprived eye) dLGN after 1 day of dark-rearing (A) demonstrate a strong monocular deprivation effect with reduced NF-H labeling in originally deprived layers (asterisks) and strong labeling in originally non-deprived layers. The boundary between dLGN layers is indicated by an arrowhead. Following 8 days of dark-rearing we observed a reduction of labeling for NF-H in both originally deprived layers as well as layers that had not been deprived (B). We have graphed our measurements of NF-H labeling and neuron size (Mean $+\mathrm{SD}$ ) in separate graphs because the

measurement of neuron size across three durations of darkrearing (Figure 3D) revealed that the size difference (deprivation metric) between deprived and non-deprived neurons observed after 1 day of dark-rearing (average $=20 \%$ ) was reduced after 4 days (average $=9 \%$ ) and by an even greater amount following 8 days of dark-rearing (average $=5 \%$ ). Following 8 days of darkrearing there was no evidence of a difference between the size of originally deprived neurons and non-deprived neurons of the dLGN. ANOVAs completed separately for each cat revealed that neurons from deprived layers were significantly smaller than nondeprived layers for all cats after 1 day $(p<0.0001)$ of recovery, for two of the four cats after 4 days ( $p<.005$; C023 and C131), but none of the cats at 8 days showed a significant difference. OneWay ANOVAs were completed separately for non-deprived and deprived layers using mean soma size and this revealed that nondeprived $\left(F_{(2,7)}<1\right)$ layers did not change significantly, whereas a significant increase was observed in deprived layers $\left(F_{(2,7)}=\right.$ $7.65, p<0.05)$, as soma size was smaller on day 1 than on days 4 and $8(p<0.05)$.

\section{DISCUSSION}

Our results show that the loss of NF-H within the dLGN that follows a period of early monocular deprivation is recoverable, and occurs pari passu with a recovery of neuron structure. Recovery of NF-H happens when either the initial deprivation is reversed, deprivation metric is not ideal due to the extremely low densities of immunopositive neurons with extended dark-rearing. Density measurements of NF-H labeling were congruent with our observations from tissue, namely that there was a reduction in labeling after dark-rearing for 4 days, and a further reduction after 8 days (C). By 8 days of dark-rearing the effect of monocular deprivation was no longer evident because of the overall reduction of labeling in originally deprived and non-deprived layers. The difference in neuron size produced by monocular deprivation, still evident after a single day of dark-rearing, was reduced after 4 days, and even further so after 8 days of dark-rearing (D). Scale bar $=100 \mu \mathrm{m}$. or when the deprived eye is opened to provide normal binocular visual input. We estimate that 8 days of either reverse occlusion or binocular vision is sufficient to promote recovery of NF-H protein to levels comparable to normal, and that both conditions enable recovery of soma size in laminae of the dLGN innervated by the initially deprived eye. In contrast to the recovery that followed introduction of visual input to the initially deprived eye, animals that were instead placed in total darkness exhibited a different pattern of results: not only did laminae in the dLGN innervated by the deprived eye show no recovery of NF-H, but additionally there was a considerable loss of NF-H labeling in laminae innervated by the non-deprived eye.

Changes in neurofilament immunoreactivity observed in the current study extend numerous previous studies that have demonstrated that proteins composing the cytoskeleton are susceptible to perturbation by various forms of abnormal visual experience. Monocular deprivation reduces labeling for NF-H in the visual system of cats (Bickford et al., 1998; Kutcher and Duffy, 2007), monkeys (Duffy and Livingstone, 2005), and humans (Duffy et al., 2007). Misalignment of the eyes (strabismus) provokes a loss of NF-H in the monkey visual cortex (Fenstemaker et al., 2001), and unilateral ocular injections of tetrodotoxin reduce levels of microtubule associated-protein 2 in deprived ocular dominance columns in monkey visual cortex (Hendry and Bhandari, 1992). In general, the anatomical effects of monocular 
deprivation have long been thought to result from a process of competition between the eyes (Wiesel and Hubel, 1965; Guillery, 1972; Hubel et al., 1977). The landmark experimental test of this idea was made by Guillery (1972) who showed that following a localized retinal lesion made in the non-deprived eye, neurons in the corresponding retinotopic region of deprived layers of the dLGN were spared the typical modification in gross structure observed elsewhere in this layer because of localized removal of the competitive advantage of the fellow eye. The reduction of NF-H after monocular deprivation also seems to require that the deprived eye be at a competitive disadvantage as opposed to a simple reduction of input (overall reduction of visually driven activity) to one eye because simultaneous closure of both eyes does not produce either a loss of NF-H (Kutcher and Duffy, 2007), a change in cell size (Guillery, 1973; Kutcher and Duffy, 2007), or a modification to the structure of geniculocortical arbors (Antonini and Stryker, 1998). The current study appears to be the first to demonstrate that deprivation-induced cytoskeleton modification in the form of neurofilament loss is recoverable under certain rearing circumstances. Restoration of an apparently normal level of NF-H labeling within originally deprived dLGN layers was observed when the competitive disadvantage that accompanies monocular deprivation was removed, either through reverse occlusion or by providing simultaneous binocular visual input. Although removal of the competitive disadvantage appears important for recovery, our findings from animals placed in complete darkness indicate that it is insufficient by itself (Figure 4). While dark-rearing, like restoration of binocular visual input, removes the competitive disadvantage of the deprived eye, it does not promote recovery of NF-H labeling. For reasons highlighted in Figure 4, we propose that recovery from monocular deprivation requires both the removal of the competitive disadvantage of the deprived eye as well as the presence of visually driven activity. Recovery occurs when the originally deprived eye is placed at an advantage (reverse occlusion) or even when the disadvantaged state is simply removed (binocular visual exposure), indicating that removal of the disadvantage is necessary. However, as indicated by the results from animals placed in darkness after monocular deprivation, it is not sufficient by itself. The importance of visually driven activity may be rooted in its ability to signal that the disadvantage created by monocular deprivation has been relieved.

Removed from the dystrophic influence of monocular deprivation, functional improvement of the vision of the deprived eye can be achieved by reverse occlusion or by providing binocular vision. In cats, the extent of the behavioral and physiological recovery achieved by either of these rearing conditions is only modestly better with reverse occlusion (Mitchell et al., 1977, 2001; Mitchell, 1988). Our anatomical results are largely in agreement with these behavioral and physiological findings by providing evidence for considerable neurofilament recovery with either recovery condition, and the observed recovery of NF-H and of neuron size was not apparently different in extent or timing across these two recovery conditions. Though the anatomical perturbations in the dLGN consequent to monocular deprivation are produced by an imbalance in competition between the eyes (Guillery, 1972), our results from binocular recovery indicate that recovery of NF-H and soma size can occur even if there is no advantage for the deprived eye. The loss of NF-H following monocular deprivation appears to require an imbalance in competition while its recovery does not, suggesting that mediating factors for these two types of plasticity are at least partly different. Additional evidence for such dissociation has been presented in previous studies of monocular deprivation and recovery. Protein synthesis (Taha and Stryker, 2002; Krahe et al., 2005), cAMP/Ca ${ }^{2+}$ response element-binding protein (Liao et al., 2002), and sleep (Dadvand et al., 2006) each have influence over the consequences of visual deprivation, but none of them seem to play a role in recovery from deprivation. However, activation of a serine protease, tissue plasminogen activator, is critical for recovery but not for the modifications

\begin{tabular}{|c|c|c|c|c|}
\hline \multirow[b]{2}{*}{ Visual Activity } & \multirow{2}{*}{ Reduced } & \multirow{2}{*}{ Reduced } & atment Condition & Dark Rearing \\
\hline & & & Normal & None \\
\hline Disadvantage & Yes & Yes & No & No \\
\hline NF-H Labeling & Loss & Recovery & Recovery & Loss \\
\hline Soma Size & Reduced & Recovery & Recovery & Recovery \\
\hline
\end{tabular}

FIGURE 4 | Schematic of the results from our investigation of NF-H labeling and neuron soma size in the dLGN across three recovery regimes (bolded text) following a period of early monocular deprivation. These results are presented alongside those from monocular lid suture from previous studies (Wiesel and Hubel, 1963a; Guillery, 1973; Kutcher and Duffy, 2007). Comparison of the effect of NF-H labeling and soma size after monocular and binocular deprivation by lid suture suggests that the loss of $\mathrm{NF}-\mathrm{H}$ and reduction in neuron size following monocular deprivation are the consequence of deprived neurons being put at a competitive disadvantage, and not because of a reduction in visually driven activity, which similarly occurs with binocular deprivation but without a reduction in NF-H (Kutcher and Duffy, 2007). Recovery of NF-H after monocular deprivation is achieved when the disadvantaged state of the deprived eye is relieved either by reverse occlusion or by restoration of binocular vision. It is noteworthy that the deprived eye need not be placed at a competitive advantage for this recovery to occur. Recovery of NF-H is evidently dependent upon visually driven activity because dark-rearing blocks recovery and leads to a pronounced overall loss of labeling. We, therefore, conclude that recovery of $\mathrm{NF}-\mathrm{H}$ requires removal of the competitive disadvantage as well as visually driven activity. The recovery of neuron soma size is also achieved when the competitive disadvantage of the deprived eye is relieved, either by reverse occlusion or provision of binocular vision, and unlike recovery of $\mathrm{NF}-\mathrm{H}$, the recovery of neuron size is evidently not dependent on visually driven activity as deprived neurons recover in complete darkness. 
initially produced by deprivation (Müller and Griesinger, 1998). The deprivation-induced loss of NF-H (Bickford et al., 1998; Duffy and Slusar, 2009) as well as its recovery likely reflect the efficacy of the experiential conditions of deprivation or recovery to initiate a cellular process that promotes some form of structural alteration that requires modification of stabilizing components of the cytoskeleton.

Neurofilaments assemble to form a stable and stationary intracellular scaffold that seems well suited for maintaining the gross structure of neurons (Morris and Lasek, 1982; Yuan et al., 2006). We have previously suggested that the structural modification of neurons following monocular deprivation is enabled partly by breakdown of stable components of the cytoskeleton such as neurofilaments (Kutcher and Duffy, 2007; Duffy and Slusar, 2009). Although the recovery of NF-H by reverse occlusion or binocular recovery occurs alongside recovery of neuron size, it is unlikely to directly mediate the growth of deprived cells because this growth occurs in the absence of recovery of NF-H during dark-rearing. The reemergence of neurofilament during recovery conditions may help to explain how stability is eventually provided to recovered neurons. The recovery stimulated by binocular vision or reverse occlusion evidently acts to bring NF-H levels back to normal either by blockade of the active processes of neurofilament removal, or perhaps by increased protein synthesis through induction of the NF-H gene silenced by monocular deprivation. Neurofilament protein has a comparatively long half-life, estimated to be between 20 and 50 days (Nixon and Logvinenko, 1986; Millecamps et al., 2007; Barry et al., 2007), and its substantial reduction after 4-6 days of monocular deprivation (Duffy and Slusar, 2009) implicates an active process at the core of its initial removal rather than a more passive process such as reduced gene expression. However, reduction in gene expression may explain why animals deprived for very long periods maintain low levels of neurofilament (Duffy and Slusar, 2009). Furthermore, while gene expression (Pham et al., 1999) and protein synthesis (Taha and Stryker, 2002) are both requirements for the functional and structural effects of monocular deprivation, recovery from such deprivation occurs independent of protein synthesis (Krahe et al., 2005). The ability of reverse occlusion and binocular vision to promote recovery of NF-H seems, therefore, unlikely to be the consequence of gene induction, and is more probably the result of blockade of an active process of removal. We previously suggested (Duffy and Slusar, 2009) that the deprivation-induced loss of neurofilament is the result of increased proteolysis provoked by the change in intracellular calcium levels that is linked to depression of synaptic efficacy observed between deprived eye cells. It is conceivable that the factors leading to the potentiation of synaptic efficacy stimulated by recovery conditions might also mitigate the cellular processes at the origin of NF-H loss.

Our quantification of the size of dLGN neurons in dark-reared kittens showed that the imbalance in neuron size produced by monocular deprivation was largely reduced following 8 days in complete darkness. Recovery of neuron size evidently requires the removal of the competitive disadvantage of the deprived eye but it does not require visually driven activity. The recovery of cell size in the absence of a recovery of NF-H highlights a dissociation that indicates postnatal cell growth can occur in the absence of a normal level of NF-H. This conclusion is supported by the observation that NF-H labeling is absent in one of the principal cell types in the dLGN, namely X neurons, (Bickford et al., 1998) despite postnatal growth. That $X$ type neurons grow independently of NF-H, and that neurons from monocularly deprived animals at one time rich with NF-H can grow in complete darkness indicates that the recovery of NF-H is not directly linked to the recovery of neuron size. Unlike recovery of NF-H, neuron size appears to recover in complete darkness and this may help to explain results that demonstrate a recovery of cortical ocular dominance in monocularly deprived cats placed in complete darkness (Freeman and Olson, 1982), and the finding (in rats) that reduced visual acuity produced by deprivation can be reversed by dark-rearing ( $\mathrm{He}$ et al., 2007). Though the absence of NF-H evidently does not limit cell growth, its presence in recovering neurons is likely to encourage the development of characteristics linked to neurofilament, including gross structural stability, large axon caliber, and fast conduction velocity (Yamasaki et al., 1992; Ohara et al., 1993; Sakaguchi et al., 1993). The link between NF-H and physiological function of the dLGN neurons that produce it, namely Y type neurons (Bickford et al., 1998), is strengthened by the observation that dark-rearing of normal animals reduces the incidence of normal physiological recordings of Y neurons without modification of the size of neurons in the dLGN (Kratz et al., 1979), and without change to the morphology of retinogeniculate Y cell axonal arbors (Garraghty et al., 1987). The loss of physiologically normal Y neurons in the dLGN raises the intriguing possibility that NF-H contributes directly to the physiological function of this neuron type, rather than indirectly through maintenance of its shape.

Loss of NF-H in the dLGN has been linked to the competitive disadvantage that is produced by monocular lid closure (Kutcher and Duffy, 2007) but we now show that NF-H is also reduced by rearing in complete darkness. While the factors that reduced NF-H in dark-reared kittens are unknown, the spontaneous retinal activity that remains in the dark may signal a reversion of maturation that includes loss of proteins, like NF-H, that are indicative of the mature state. Evidence of developmental recapitulation has been shown to occur in the monkey somatosensory cortex with introduction of median nerve compression (Mowery and Garraghty, 2009; Mowery et al., 2011), and in the auditory cortex of rats exposed to white noise there is evidence for restoration of critical period plasticity (Zhou et al., 2011). The loss of NF-H with dark-rearing could initially be interpreted as evidence for a severe processing impairment given the importance of $\mathrm{NF}-\mathrm{H}$ for maintenance of axon caliber and fast conduction velocity (Yamasaki et al., 1992; Ohara et al., 1993; Sakaguchi et al., 1993). Indeed the reduction of NF-H may contribute to visual system dysfunction that results from a period of dark-rearing (Blakemore and Van Sluyters, 1975; Buisseret and Imbert, 1976; Mower et al., 1981; Blakemore and Price, 1987). However, in the context of monocular deprivation, where one eye's anatomical and physiological characteristics are deeply abnormal, imposition of periods of darkness could provide a means to reduce eye-specific differences prior to introduction of normal visual experience and as a consequence enhance recovery. Indeed, it has been shown recently that monocularly deprived rats placed in 
darkness for 10 days prior to reverse occlusion exhibit recovery of dendritic spines throughout visual cortex (Montey and Quinlan, 2011). The observation (He et al., 2007) of enhanced functional recovery of the deprived eye following a 10 day period of darkness imposed on adult rats supports the conjecture that the latter experiential manipulation may trigger cellular events that subsequently allow functional recovery. More importantly, preliminary results (Duffy and Mitchell, in preparation) of the effects of a 10 day period of darkness imposed on monocularly deprived kittens indicate that the latter can promote both rapid and substantial recovery of the vision of the deprived eye. An improvement

\section{REFERENCES}

Antonini, A., and Stryker, M. P. (1993). Rapid remodeling of axonal arbors in the visual cortex. Science 260, 1819-1821.

Antonini, A., and Stryker, M. P. (1998). Effect of sensory disuse on geniculate afferents to cat visual cortex. Vis. Neurosci. 15, 401-409.

Barry, D. M., Millecamps, S., Julien, J.-P., and Garcia, M. L. (2007). New movements in neurofilament transport, turnover and disease. Exp. Cell Res. 313, 2110-2120.

Beaver, C. J., Mitchell, D. E., and Robertson, H. A. (1993). Immunohistochemical study of the patter of rapid expression of $\mathrm{C}$-Fos protein in the visual cortex of dark-reared kittens following initial exposure to light. J. Comp. Neurol. 333, 469-484.

Bickford, M. E., Guido, W., and Godwin, D. W. (1998). Neurofilament proteins in Y-cells of the cat lateral geniculate nucleus: normal expression and alteration with visual deprivation. J. Neurosci. 18, 6549-6557.

Blakemore, C., and Van Sluyters, R. C. (1975). Innate and environmental factors in the development of the kitten's visual cortex. J. Physiol. 248, 663-716.

Blakemore, C., and Price, D. J. (1987). Effects of dark-rearing on the development of area 18 of the cat's visual cortex. J. Physiol. 384, 294-309.

Buisseret, P., and Imbert, M. (1976). Visual cortical cells: their developmental properties in normal and dark reared kittens. J. Physiol. 255, 511-525.

Dadvand, L., Stryker, M. P., and Frank, M. G. (2006). Sleep does not enhance the recovery of deprived eye responses in developing visual cortex. Neuroscience 143, 815-826.

Daw, N. W. (2006). Visual Development, 2nd edn. New York, NY: Springer.

Dews, P. B., and Wiesel, T. N. (1970). Consequences of monocular deprivation on visual behaviour in kittens. J. Physiol. 206, 437-455.
Duffy, K. R., Crowder, N. A., and LeDue, E. E. (2011). Investigation of cytoskeleton proteins in neurons of the cat lateral geniculate nucleus. J. Comp. Neurol. 520, 186-199.

Duffy, K. R., and Livingstone, M. S. (2005). Loss of neurofilament labeling in the primary visual cortex of monocularly deprived monkeys. Cereb. Cortex 15, 1146-1154.

Duffy, K. R., Murphy, K. M., Frosch, M. P., and Livingstone, M. S. (2007). Cytochrome oxidase and neurofilament reactivity in monocularly deprived human visual cortex. Cereb. Cortex 17, 1283-1791.

Duffy, K. R., and Slusar, J. E. (2009). Monocular deprivation provokes alteration of the neuronal cytoskeleton in developing cat lateral geniculate nucleus. Vis. Neurosci. 26, 319-328.

Dürsteler, M. R., Garey, L. J., and Movshon, J. A. (1976). Reversal of the morphological effects of monocular deprivation in the kitten's lateral geniculate nucleus. J. Physiol. 261, 189-210.

Fava, M. A., Duffy, K. R., and Murphy, K. M. (1999). Experience-dependent development of NMDAR1 subunit expression in the lateral geniculate nucleus. Vis. Neurosci. 16, 781-789.

Fenstemaker, S. B., Kiorpes, L., and Movshon, J. A. (2001). Effects of experimental strabismus on the architecture of macaque monkey striate cortex. J. Comp. Neurol. 438, 300-317.

Frank, M. G., Issa, N. P., and Stryker, M. P. (2001). Sleep enhances plasticNeuron 30, 275-287.

Freeman, R. D., and Olson, C. (1982). Brief periods of monocular deprivation in kittens: effects of delay prior to physiological study. J. Neurophysiol. 47, 139-150.

Garey, L. J., and Vital-Durand, F. (1981). Recovery from monocular deprivation in the monkey. II. Reversal of morphological effects in the lateral ity in the developing visual cortex.

in the efficacy of treatment for amblyopia through dark-rearing may originate from enhanced structural flexibility consequent to a generalized loss of structure-providing proteins such as neurofilament.

\section{ACKNOWLEDGMENTS}

The authors thank Jan Kennie for assistance in data collection. This work was supported by grants from the Natural Sciences and Engineering Research Council of Canada (Kevin R. Duffy and Donald E. Mitchell), and by a grant from the Canadian Institutes of Health Research (Kevin R. Duffy and Donald E. Mitchell).

geniculate nucleus. Proc. R. Soc. Lond. B Biol. Sci. 213, 425-433.

Garraghty, P. E., Frost, D. O., and Sur, M. (1987). The morphology of retinogeniculate $\mathrm{X}$ - and $\mathrm{Y}$-cell axonal arbors in dark-reared cats. Exp. Brain Res. 66, 115-127.

Georges, E., and Mushynski, W. E. (1987). Chemical modification of charged amino acid moieties alters the electrophoretic mobilities of neurofilament subunits on SDS/polyacrylamide gels. Eur. J. Biochem. 165, 281-287.

Giffin, F., and Mitchell, D. E. (1978). The rate of recovery of vision after early monocular deprivation in kittens. J. Physiol. 274, 511-537.

Guillery, R. W. (1972). Binocular competition in the control of geniculate cell growth. J. Comp. Neurol. 144, 117-130.

Guillery, R. W. (1973). The effect of lid suture upon the growth of cell in the dorsal lateral geniculate nucleus of kittens. J. Comp. Neurol. 148, 417-422.

Guillery, R. W., and Stelzner, D. J. (1970). The differential effects of unilateral lid closure upon the monocular and binocular segments of the dorsal lateral geniculate nucleus in the cat. J. Comp. Neurol. 139, 413-421.

Harwerth, R. S., Smith, E. L. 3rd., Boltz, R. L., Crawford, M. L. J., and von Noorden, G. K. (1983). Behavioral studies on the effect of abnormal early visual experience in monkeys: spatial modulation sensitivity. Vis. Res. 23, 1501-1510.

He, H. Y., Ray, B., Dennis, K., and Quinlan, E. M. (2007). Experiencedependent recovery of vision following chronic deprivation amblyopia. Nat. Neurosci. 10, 1134-1136.

Hendry, S. H., and Bhandari, M. A. (1992). Neuronal organization and plasticity in adult monkey visual cortex: immunoreactivity for microtubule-associated protein 2 . Vis. Neurosci. 9, 445-459.

Heynen, A. J., Yoon, B. J., Liu, C. H., Chung, H. J., Huganir, R. L., and Bear, M. F. (2003). Molecular mechanism for loss of visual cortical responsiveness following brief monocular deprivation. Nat. Neurosci. 6, 854-862.

Hubel, D. H., Wiesel, T. N., and LeVay, S. (1977). Plasticity of ocular dominance columns in monkey striate cortex. Philos. Trans. R. Soc. Lond. B Biol. Sci. 278, 377-409.

Julien, J. P., and Mushynski, W. E. (1982). Multiple phosphorylation sites in mammalian neurofilament polypeptides. J. Biol. Chem. 257, 10467-10470.

Kaufmann, E., Geisler, N., and Weber, K. (1984). SDS-PAGE strongly overestimates the molecular masses of the neurofilament proteins. FEBS Lett. 170, 81-84.

Kossut, M., Thompson, I. D., and Blakemore, C. (1983). Ocular dominance columns in cat striate cortex and effects of monocular deprivation: a 2-deoxyglucose study. Acta Neurobiol. Exp. (Wars) 43, 273-282.

Krahe, T. E., Medina, A. E., de Bittencourt-Navarrete, R. E. Colello, R. J., and Ramoa, A. S. (2005). Protein synthesisindependent plasticity mediates rapid and precise recovery of deprived eye responses. Neuron 48, 329-343.

Kratz, K. E., Sherman, S. M., and Kalil, R. (1979). Lateral geniculate nucleus in dark-reared cats: loss of Y cells without changes in cell size. Science 203, 1353-1355.

Kutcher, M. R., and Duffy, K. R. (2007). Cytoskeleton alteration correlates with gross structural plasticity in the cat lateral geniculate nucleus. Vis. Neurosci. 24, 775-785.

LeVay, S., Wiesel, T. N., and Hubel, D. H. (1980). The development of ocular dominance columns in normal and visually deprived monkeys. J. Comp. Neurol. 191, 1-51.

Liao, D. S., Mower, A. F., Neve, R. L., Sato-Bigbee, C., and Ramoa, A. S. (2002). Different mechanisms for loss and recovery of binocularity in 
the visual cortex. J. Neurosci. 22, 9015-9023.

Mataga, N., Mizuguchi, Y., and Hensch, T. K. (2004). Experience-dependent pruning of dendritic spines in visual cortex by tissue plasminogen activator. Neuron 44, 1031-1041.

Millecamps, S., Gowing, G., Corti, O., Mallet, J., and Julien, J.-P. (2007). Conditional NF-L transgene expression in mice for in vivo analysis of turnover and transport rate of neurofilaments. J. Neurosci. 27, 4947-4956.

Mioche, L., and Singer, W. (1989). Chronic recordings from single sites of kitten striate cortex during experience-dependent modifications of receptive-field properties. J. Neurophysiol. 62, 185-197.

Mitchell, D. E. (1988). The extent of visual recovery from early monocular or binocular visual deprivation in kittens. J. Physiol. 395, 639-660.

Mitchell, D. E., Cynader, M., and Movshon, J. A. (1977). Recovery from the effects of monocular deprivation in kittens. J. Comp. Neurol. 176, 53-63.

Mitchell, D. E., Gingras, G., and Kind, P. C. (2001). Initial recovery of vision after early monocular deprivation in kittens is faster when both eyes are open. Proc. Natl. Acad. Sci. U.S.A. 98, 11662-11667.

Montey, K. L., and Quinlan, E. M. (2011). Recovery from chronic monocular deprivation following reactivation of thalamocortical plasticity by dark exposure. Nat. Commun. 2, 317.

Morris, J. R., and Lasek, R. J. (1982). Stable polymers of the axonal cytoskeleton: the axoplasmic ghost. J. Cell Biol. 92, 192-198.

Movshon, J. A. (1976a). Reversal of the behavioural effects of monocular deprivation in kittens. J. Physiol. 261, 175-187.

Movshon, J. A. (1976b). Reversal of the physiological effects of monocular deprivation in the kitten's visual cortex. J. Physiol. 261, 125-174.

Mower, G. D., Burchfiel, J. L., and Duffy, F. H. (1981). The effects of dark-rearing on the development and plasticity of the lateral geniculate nucleus. Brain Res. 227, 418-424.

Mowery, T. M., and Garraghty, P. E. (2009). Nerve-injury induced changes to GluR1 and GluR2/3 subunit expression in area $3 \mathrm{~b}$ of adult squirrel monkeys: developmental recapitulation? Front. Syst. Neurosci. 3:1. doi: 10.3389/neuro.06.001.2009

Mowery, T. M., Sarin, R. M., Elliott, K. S., and Garraghty, P. E. (2011). Nerve injury-induced changes in GABAA and GABAB sub-unit expression in area $3 \mathrm{~b}$ and cuneate nucleus of adult squirrel monkeys: further evidence of developmental recapitulation. Brain Res. 1415, 63-75.

Müller, C. M., and Griesinger, C. B. (1998). Tissue plasminogen activator mediates reverse occlusion plasticity in visual cortex. Nat. Neurosci. 1, 47-53.

Murphy, K. M., and Mitchell, D. E. (1987). Reduced visual acuity in both eyes of monocularly deprived kittens following a short or long period of reverse occlusion. J. Neurosci. 7, 1526-1536.

Nixon, R. A., and Logvinenko, K. B. (1986). Multiple fates of newly synthesized neurofilament proteins: evidence for a stationary neurofilament network distributed nonuniformly along axons of retinal ganglion cell neurons. J. Cell Biol. 102, 647-659.

Ohara, O., Gahara, Y., Miyake, T., Teraoka, H., and Kitamura, T. (1993). Neurofilament deficiency in quail caused by nonsense mutation in neurofilament-L gene. J. Cell Biol. 121, 387-395.

Olson, C. R., and Freeman, R. D. (1978). Monocular deprivation and recovery during sensitive period in kittens. J. Neurophysiol. 41, 65-74.

Olson, C. R., and Freeman, R. D. (1980). Profile of the sensitive period for monocular deprivation in kittens. Exp. Brain Res. 39, 17-21.

Pham, T. A., Impey, S., Storm, D. R. and Stryker, M. P. (1999). CREmediated gene transcription in neocortical neuronal plasticity during the developmental critical period. Neuron 22, 63-72.

Sakaguchi, T., Okada, M., Kitamura, T. and Kawasaki, K. (1993). Reduced diameter and conduction velocity of myelinated fibers in the sciatic nerve of a neurofilament-deficient mutant quail. Neurosci. Lett. 153, 65-68.

Sanderson, K. J. (1971). The projection of the visual field to the lateral geniculate and medial interlaminar nuclei in the cat. J. Comp. Neurol. $143,101-118$.

Sato, M., and Stryker, M. P. (2008). Distinctive features of adult ocular dominance plasticity. J. Neurosci. 28, 10278-10286.

Taha, S., and Stryker, M. P. (2002). Rapid ocular dominance plasticity requires cortical but not geniculate protein synthesis. Neuron 34 425-436.

Tremain, K. E., and Ikeda, H. (1982). Relationship between amblyopia, LGN cell "shinkage" and cortical ocular dominance in cats. Exp. Brain Res. 45, 243-252.

Wiesel, T. N., and Hubel, D. H. (1963a) Single-cell responses in striate cortex of kittens deprived of vision in one eye. J. Neurophysiol. 26, 1003-1017.

Wiesel, T. N., and Hubel, D. H. (1963b). Effects of visual deprivation on morphology and physiology of cells in the cats lateral geniculate body. $J$. Neurophysiol. 26, 978-993.

Wiesel, T. N., and Hubel, D. H. (1965). Comparison of the effects of unilateral and bilateral eye closure on cortical unit responses in kittens. J. Neurophysiol. 28, 1029-1040.

Yamasaki, H., Bennett, G. S., Itakura, C., and Mizutani, M. (1992). Defective expression of neurofilament protein subunits in hereditary hypotrophic axonopathy of quail. Lab. Invest. 66, 734-743.

Yuan, A., Rao, M. V., Sasaki, T., Chen, Y., Kumar, A., Veeranna, Liem, R. K. H., Eyer, J., Peterson, A. C., Julien, J.-P., and Nixon, R. A. (2006). A-internexin is structurally and functionally associated with the neurofilament triplet proteins in the mature CNS. J. Neurosci. 26, 10006-10019.

Zhou, X., Panizzutti, R., de VillersSidani, E., Madeira, C., and Merzenich, M. M. (2011). Natural restoration of critical period plasticity in the juvenile and adult primary auditory cortex. J. Neurosci. 31, 5625-5634.

Conflict of Interest Statement: The authors declare that the research was conducted in the absence of any commercial or financial relationships that could be construed as a potential conflict of interest.

Received: 03 January 2012; accepted: 17 March 2012; published online: 09 April 2012.

Citation: O'Leary TP, Kutcher $M R$, Mitchell DE, and Duffy KR (2012) Recovery of neurofilament following early monocular deprivation. Front. Syst. Neurosci. 6:22. doi: 10.3389/fnsys. 2012.00022

Copyright (C) 2012 O'Leary, Kutcher, Mitchell and Duffy. This is an openaccess article distributed under the terms of the Creative Commons Attribution Non Commercial License, which permits non-commercial use, distribution, and reproduction in other forums, provided the original authors and source are credited. 